\title{
Three-dimensional Chained Nonholonomic Systems Stabilization Control via Dynamic Feedback
}

\author{
Ting-ting Wang, Tian-you Li and Wan-chun Zhao* \\ Northeast Petroleum University, Daqing 163318, China \\ E-mail:wttlovework@163.com
}

\begin{abstract}
Three kinds of global universal controllers are proposed for nonholonomic systems, namely, the universal exponential regulators, the universal $K$-exponential controller and the universal practical controller. With help of an introduced state and the dynamic feedback technique, a controller with special structure is constructed to obtain an augmented closed-loop error system. The error system tends to continuous oscillation. So the controller structure is modified to loosen the control objective to practical stability and the error can converge to a neighborhood of origin as small as possible. Thus, oscillation and peaking phenomena are avoided and engineering precision is obtained. Detailed simulations on the three-dimension chained system are carried out, and the results show the effectiveness of the proposed controllers.
\end{abstract}

Keywords: Nonholonomic systems; Universal Controller; Point Stabilization

\section{Introduction}

In recent years, with the development of the mobile robot technology and aerospace technology, the control problems of nonholonomic systems get the attention of the scholars around the world. Because the constraint equations of this kind of system always contain differential terms and nonholonomic constraint equation [1], it's called the nonholonomic system.

In engineering practice, there are a lot of nonholonomic systems, the causes of this phenomenon are various, some of which are caused due to the moving characteristics of the object itself, such as for pure roll, no sliding movement of the ball and sliding on the ice skate, wheeled mobile robot [2-4], etc.; Also some of them considers actual situation and are artificially added, such as flexible manipulators[5], intelligent vehicle [6], the surface ship [7], etc.; Some of them are caused by under-actuated, such as satellites in space and the space shuttle, space robot [8], etc. These systems have common characteristics: they are constrained by the nonholonomic constraint equation, namely in the constraint equations of the nonholonomic system contain at least one differential item, and unintegrable constraint equation. Because the approximate linearization of nonholonomic systems are uncontrolled, the continuous static state feedback control law cannot be applied to realize the whole state of stabilization [9-10]. It has important theoretical significance for noholonomic system control.

Based on such typical nonholonomic wheeled mobile robot system to study the point stabilization and trajectory tracking control problem, when the control task switching between point stabilization and trajectory tracking, require the system to switch to the corresponding controller, and this kind of switch is likely to make the system produce shock, the components damage [11-13]. In order to avoid this kind of switch, scholars put forward the unified controller, the controller of processing a variety of control tasks at the same time. 
At present, the main content of the control problem of nonholonomic systems research including object model establishment and transform, motion planning, fixed point stabilization and trajectory tracking and optimization control and so on.

Many industrial controls contain both fixed point stabilization and trajectory tracking [14-18]. Traditional switcher will cause the following problems:

(1) Switching can cause the shock, it is harm to the system;

(2) It is hard to determine the switch time exactly, the delay features of the practical system will cause the system worsen. It is necessary to study and apply to the unity of the fixed point stabilization and trajectory tracking controller.

The content of reference [19] introduce the dynamic feedback technology (but not for the linearization) for the second order nonholonomic system, the global continuous time invariant dynamic feedback controller is designed, without any switching, obtained the exponential convergence speed, but didn't get the asymptotic stability, when $t \rightarrow \infty$, systems tend to be more persistent oscillation.

According to the content of reference [20], a small constant $\delta>0$ can be introduced for three-dimensional chain systems in order to improve the structure of controller except any switches [21-22]. It can eliminate persistent oscillation and obtain the exponential of convergence speed.

\section{Global Universal Control}

\subsection{Universal Exponential Regulator}

The mathematical model of three-dimension chain systems is as follows:

$$
\dot{x}_{1}=u_{1}, \dot{x}_{2}=u_{2}, \dot{x}_{3}=x_{2} u_{1}
$$

Where $X=\left[x_{1}, x_{2}, x_{3}\right]^{T}$ is the system state, $\left(u_{1}, u_{2}\right)$ is control input. The reference mode of system (1) is

$$
\dot{x}_{1 r}=u_{1 r}, \dot{x}_{2 r}=u_{2 r}, \dot{x}_{3 r}=x_{2 r} u_{1 r}
$$

Where $X_{r}=\left[x_{1 r}, x_{2 r}, x_{3 r}\right]^{T}$ is the system state, $\left(u_{1 r}, u_{2 r}\right)$ is control input.

Where $X_{r}=\left[x_{1 r}, x_{2 r}, x_{3 r}\right]^{T}$ is the system state, $\left(u_{1 \mathrm{r}}, u_{2 r}\right)$ is control input.

\subsection{Universal K-exponential Controller}

To obtain asymptotic stability, choose $\mathrm{K}$-class function $k_{0}(o)$ as the initial value of auxiliary dynamic variables $x_{2 d}$, where $o \square\left\|\boldsymbol{X}_{e}(0)\right\|$.

Theorem 1 If the system is $k_{2}>2 k_{1}>0, k_{3}>k_{1}$, the initial value of $x_{2 d}$ is

$$
x_{2 d}(0)=\sqrt{-x^{2}{ }_{1 \varepsilon}(0)+\sqrt{x^{4}{ }_{1 \varepsilon}(0)+o^{4 \varepsilon}}} \leq o^{\varepsilon}, 0<\varepsilon<1
$$

The continuous control law is as follows:

$$
\begin{aligned}
& x_{e}(0)=0, u_{1}=u_{1 r}, u_{2}=u_{2 r} \\
& u_{1}=u_{1 r}-k_{1} x_{1 e}-k_{3} x_{2 d} z / V \\
& u_{2}=u_{2 r}+\dot{x}_{2 d}-k_{2} e_{2} \\
& \dot{x}_{2 d}=-k_{1} x_{2 d}+k_{3} x_{1 e} z / V
\end{aligned}
$$

$\left(u_{1}, u_{2}\right)$ is in bounded and the $X_{\varepsilon}$ is $\mathrm{K}$ global exponential stability.

\section{Demonstrate}

1) When $X_{\varepsilon}(0)=0, X_{\varepsilon}(t)=0(\forall t \geq 0)$, so $\left(u_{1}, u_{2}\right)$ is in bounded. 
2) Suppose $\boldsymbol{X}_{e}(0) \neq 0$.By the equation (3) and the $a^{2}+b^{2} \leq(a+b)^{2}$ (if $a b>0$ ) we known,

$$
\begin{gathered}
V(0)=\frac{1}{2}\left(x_{1 s}^{2}(0)+x_{2 d}^{2}(0)\right) \leq \frac{1}{2}\left(o+o^{s}\right)^{2} \\
V(0)=\frac{1}{2}\left(x_{1 s}^{2}(0)+x_{2 d}^{2}(0)\right)=\frac{1}{2} \sqrt{x_{1 s}^{4}(0)+o^{4 s}} \geq \frac{1}{2} o^{2 s}
\end{gathered}
$$

For short, define $O=o+o^{\varepsilon}$, time derivative of the closed-loop system $V$ satisfies:

$$
\begin{aligned}
& \dot{V}=-2 k_{1} V \Rightarrow 0<V(t)=V(0) e^{-2 k_{1} t} \\
& \Rightarrow \max \left(\left|x_{1 \varepsilon}(t)\right|,\left|x_{2 d}(t)\right|\right) \leq \sqrt{2 V(t)} \leq O e^{-2 k_{1} t}
\end{aligned}
$$

From equation (3) and $k_{2}>2 k_{1}$,

$$
\left|e_{2}(t)\right|=\left|e_{2}(0)\right| e^{-k_{2} t}=\left|x_{2 \varepsilon}(0)-x_{2 d}(0)\right| e^{-k_{2} t} \leq O e^{-2 k_{1} t}
$$

From equation (7) and (8),

$$
\left|x_{2 \varepsilon}(t)\right| \leq\left|e_{2}(t)\right|+\left|x_{2 d}(t)\right| \leq 2 O e^{-k_{1} t}
$$

According to the equation $h=e_{2} x_{2 d} / V$ and equation (6)-(9),

$$
\begin{array}{r}
\left|e_{2}(t)\right| \max \left(\left|x_{1 \varepsilon}(t),\right| x_{2 d}(t) \mid\right) \leq O^{2} e^{-3 k_{1} t} \\
|h(t)| \leq O^{2} e^{-3 k_{1} t} /\left(\frac{1}{2} o^{2 \varepsilon} e^{-2 k_{1} t}\right) \leq 2\left(o^{1-\varepsilon}+1\right) e^{-k_{1} t}
\end{array}
$$

There exists strict increasing function $c_{2}(o)$ which satisfies

$$
\lim _{t \rightarrow \infty}|h(t)|=0, \int_{0}^{\infty}|h(t)| d t \leq c_{2}(o)
$$

According to the definition $f$ and equation from (7)-(10),

$$
\begin{aligned}
& |f| \leq\left|x_{2 d} u_{1 r}\right|+2\left|x_{1 \varepsilon} u_{2 r}\right|+\left(k_{2}-k_{1}\right)\left|e_{2} x_{1 \varepsilon}\right|+\left(\left|e_{2}\right|+\left|x_{2 \varepsilon}\right|\right)\left|u_{1 r}\right| \\
& \leq 3 M O e^{-k_{1} t}+\left(k_{2}-k_{1}\right) O^{2} e^{-3 k_{1} t}+M O\left(e^{-k_{1} t}+2\right) e^{-k_{1}} \\
& \leq k_{0}(o) e^{-k_{1} t}
\end{aligned}
$$

Where $\kappa_{0}(o)=6 M O+\left(k_{2}-k_{1}\right) O^{2}$ is K-class function. By the definition of $z$, we know:

$$
\begin{aligned}
& |z(0)| \leq 2\left|x_{3 \varepsilon}(0)\right|+\left|x_{1 \varepsilon}(0)\right|\left|x_{2 \varepsilon}(0)\right|+2\left|x_{2 \gamma}(0)\right|\left|x_{1 \varepsilon(0)}\right| \\
& \leq 2 o+2\left|x_{2 \gamma}(0)\right| o+o^{2}
\end{aligned}
$$

Consider the fourth system equation, both $f$ and $h$ converge to zero with the exponential of $k_{1}$ and $k_{2}-k_{1}$ respectively.

From equation (12)-(14), there is constant $\left(c_{4}, c_{5}, c_{6}\right)$ satisfies the following equation:

$$
|z(t)| \leq\left(c_{4}|z(0)|+c_{5} \kappa_{0}(0)\right) e^{c_{6} c_{2}(0)} e^{-\kappa_{1} t}
$$

Substitute equation (14) to the above equation, there is the K-class function $\kappa_{1}(\cdot)$ satisfies

$$
|z(t)| \leq \kappa_{1}(o) e^{-\kappa_{1} t}
$$

Where $\left.\kappa_{1}(o)=\mid c_{4}\left(2 o+2\left|x_{2 r}(0)\right| o+o^{2}\right)+c_{5} \kappa_{0}(o)\right] e^{c_{6} c_{2}(o)}$ is K-class function.

From the equation (7),

$$
\left.\left|x_{2 r}(t) x_{1 \varepsilon}(t)\right| \leq\left(M t+\mid x_{2 r}(0)\right)\right) O e^{-\kappa_{1} t} \leq\left(c_{3}+\left|x_{2 r}(0)\right|\right) O e^{-\kappa_{1} t}
$$

According to the definition of $\mathrm{z}$, and equation (7), (9), (15), (16), there is the following equation: 


$$
\begin{aligned}
& \left|x_{3 e}(t)\right| \leq \frac{1}{2}\left(|z(t)|+\left|x_{1 \varepsilon}(t)\right|\left|x_{2 \varepsilon}(t)\right|+2\left|x_{2 r}(t) x_{1 \varepsilon}(t)\right|\right) \\
& \leq \frac{1}{2}\left(\kappa_{1}(o) e^{-\kappa_{1} t}+O^{2} e^{-2 \kappa_{1} t}+2\left(c_{3}+\left|x_{2 r}(0)\right| O e^{-\kappa_{4} t}\right)\right) \\
& \leq \kappa_{2}(o) e^{-\kappa_{4} t}
\end{aligned}
$$

Where $\kappa_{2}(o)=\frac{1}{2}\left[\kappa_{1}(o)+O^{2}+2\left(c_{3}+\left|x_{2 r}(0)\right|\right) o\right]$ is K-class function.

From equation (7), (9) and (17), there is

$$
\left\|X_{\varepsilon}(t)\right\| \leq\left|x_{1 \varepsilon}(t)\right|+\left|x_{2 \varepsilon}(t)\right|+\left|x_{3 \varepsilon}(t)\right| \leq \kappa_{3}(o) e^{-\kappa_{4} t}
$$

Where $\kappa_{3}(o)=3 O+\kappa_{2}(o)$ is K-class function. So $X_{e}(t)$ is K-exponential stable.

3) Suppose $\boldsymbol{X}_{e}(0) \neq 0$, to prove $\left(u_{1}, u_{2}\right)$ is in bounded. By the equation (4),(6),(7), (15),

$$
\begin{aligned}
& \left|u_{1}(t)\right| \leq\left|u_{1}(t)\right|+k_{1}\left|x_{1 \varepsilon}(t)\right|+k_{1}\left|x_{2 d}(t)\right| z(t) \mid / V \\
& \leq M+k_{1} O e^{-k_{1} t}+\frac{k_{1} O e^{-k_{1} t} \kappa_{1}(o) e^{-k_{1} t}}{\frac{1}{2} o^{2 \delta} e^{-2 k_{1} t}}
\end{aligned}
$$

Above all, both $O$ and $\kappa_{1}(o)$ are the same order infinitesimal of $o^{\varepsilon}$.So $\left|u_{1}(t)\right|$ is in bounded. So $\left|\dot{x}_{2 d}(t)\right|$ is in bounded in the same way, and $\left|u_{2}(t)\right|$ is also in bounded.

By the way, $\left(u_{1}, u_{2}\right)$ will not converge to zero except fixed-point stabilization.

4) Suppose $\boldsymbol{X}_{e}(0) \neq 0$, to prove $\left(u_{1}, u_{2}\right)$ is continuous. According to equation (1)-(3), only have to prove that when $\boldsymbol{X}_{e}(0) \rightarrow 0(o \rightarrow 0)$, there is $\left(u_{1}, u_{2}\right) \rightarrow\left(u_{1 r}, u_{2 r}\right)$. From definition $\mathrm{z}$ there is the following equation:

$$
\begin{aligned}
& \lim _{o \rightarrow 0} z=2 x_{3 \varepsilon}(0)-x_{1 \varepsilon}(0) x_{2 \varepsilon}(0)-2 x_{2 r}(0) x_{1 \varepsilon}(0) \\
& \Rightarrow\left|\lim _{o \rightarrow 0} z\right| \leq\left(2+\left|x_{2 \varepsilon}(0)\right|+2\left|x_{2 r}(0)\right|\right) o
\end{aligned}
$$

From (6) and (14), there is the following equation:

From (4), it can obtain:

$$
\left|\lim _{o \rightarrow 0} x_{2 d}(0) z(0) / V(0)\right| \leq \lim _{o \rightarrow 0} o^{\delta}\left(2+\left|x_{2 \varepsilon}(0)\right|+2\left|x_{2 r}(0)\right|\right) o /\left(\frac{1}{2} o^{2 \delta}\right)
$$

$$
\lim _{o \rightarrow 0} u_{1}(0)=u_{1 r}(0)-\lim _{o \rightarrow 0}\left(k_{1} x_{1 \varepsilon}(0)+k_{3} x_{2 d}(0) z(0) / V(0)\right)=u_{1 r}(0)
$$

So $u_{1}$ is continuous. It can be proved that $u_{2}$ is also continuous in the same way.

\subsection{Universal Practical Controller}

For universal practical control, the system tracking error converges to a small neighborhood of origin. Because the neighborhood can be arbitrarily small, therefore, for the engineering application, it can ensure accuracy.

Theorem 2 If $k_{2}>2 k_{1}>0, k_{3}>k_{1}$, error bound is $\delta>0$, choose $x_{2 d}(0) \neq 0$, from the smooth control law:

$$
\left\{\begin{array}{c}
u_{1}=u_{1 r}-k_{1} x_{1 \varepsilon}-k_{3} x_{2 d} z /\left(V+k_{4}\right) \\
u_{2}=u_{2 r}+\dot{x}_{2 d}-k_{2} e_{2} \\
\dot{x}_{2 d}=-k_{1} x_{2 d}+k_{3} x_{1 \varepsilon} z /\left(V+k_{4}\right)
\end{array}, 0 \leq k_{4} \leq\left(2 k_{3}-k_{1}\right) \delta / k_{1}\right.
$$

State trajectory $\boldsymbol{X}_{e}(t)$ is ultimately global bounded, control input $\left(u_{1}, u_{2}\right)$ is in bounded,. $\lim _{t \rightarrow \infty}\left(u_{1}(t), u_{2}(t)\right)=\left(u_{1 r}(t), u_{2 r}(t)\right)$

Demonstrate A closed loop system is as follows: 


$$
\left\{\begin{array}{c}
\dot{x}_{1 \varepsilon}=-k_{1} x_{1 \varepsilon}-k_{3} x_{2 d} z /\left(V+k_{4}\right) \\
\dot{x}_{2 d}=-k_{1} x_{2 d}-k_{3} x_{1 \varepsilon} z /\left(V+k_{4}\right) \\
\dot{e}_{2}=-k_{2} e_{2} \\
\dot{z}=-2 k_{3} V z /\left(V+k_{4}\right)-k_{3} h z+f
\end{array}\right.
$$

Where $h=e_{2} x_{2 d} /\left(V+k_{4}\right), V$ and $f$ are defined in (9) and (10) respectively, time derivative $\dot{V}$ satisfies $\dot{V}=-2 k_{1} V \Rightarrow V(t)=V(0) e^{-2 k_{1} t}$, so $\left(x_{1 \varepsilon}(t), x_{2 d}(t)\right)$ converges to zero with the speed of $k_{1}$ exponential. Finally, consider the definition of $z,\left|x_{2 r}(t) x_{1 \varepsilon}(t)\right|$ is exponential convergence, in the same way, it is practical stability.

Because $V+\delta$ has lower bound, so both $x_{2 d} z /\left(V+k_{4}\right)$ and $x_{1 \varepsilon} z /\left(V+k_{4}\right)$ can converge to zero, $\left(u_{1}, u_{2}\right)$ is in bounded, and $\lim _{t \rightarrow \infty}\left(u_{1}(t), u_{2}(t)\right)=\left(u_{1 r}(t), u_{2 r}(t)\right)$.

From demonstrate of Theorem 2, because the numerator of $x_{2 d} z /\left(V+k_{4}\right)$ and $x_{1 \varepsilon} z /\left(V+k_{4}\right)$ are both exponential convergent, the denominator has lower bound $(\delta>0)$, so it avoid the peaking phenomenon in the theorem 1 completely.

\section{The Simulation Verification}

Considering the unicycle mobile robot kinematics model as follows:

$$
\dot{x}=v \cos \theta, \dot{y}=v \sin \theta, \dot{\theta}=\omega
$$

From a simple transformation,

$$
\begin{aligned}
& x_{1}=\theta, x_{2}=x \cos \theta+y \sin \theta, x_{3}=y \sin \theta-x \cos \theta \\
& u_{1}=\omega, u_{2}=v-\omega x_{3}
\end{aligned}
$$

It can obtain a three-dimension chain system $\dot{x}_{1}=u_{1}, \dot{x}_{2}=u_{2}, \dot{x}_{3}=x_{2} u_{1}$.

Suppose that the system initial state is $(x, y, \theta)=(-1,-1, \pi / 4)$, initial state of reference system is $\left(x_{r}, y_{r}, \theta_{r}\right)=(0,0,-\pi / 6)$. Choose the controller parameters as

$$
k_{1}=0.2, k_{2}=2, k_{3}=1.5, k_{4}=1.4, \delta=0.1 \text {. }
$$

\subsection{Straight Line Tracking}

Target trajectory is a straight line, using the controller mentioned in Theorem 2. The results of the simulation are shown in Figure 1, the controller mentioned in this section can track the reference line better and faster. 


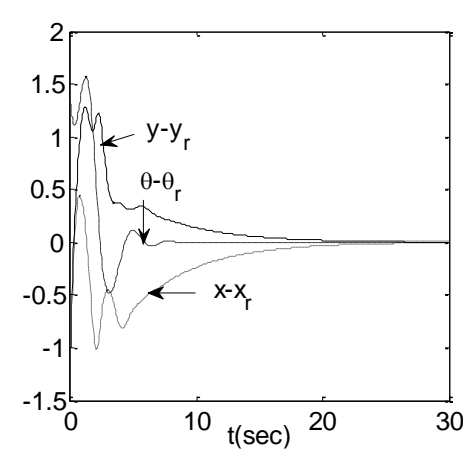

(a) State Error

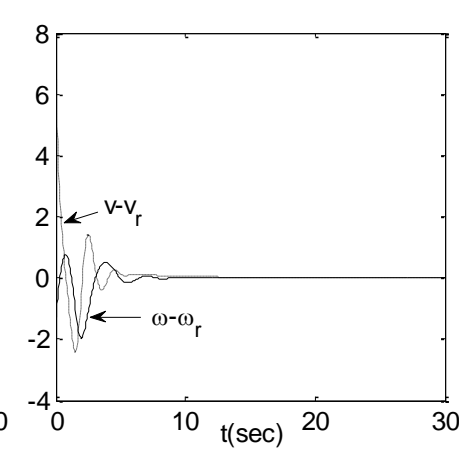

(b) Control Error

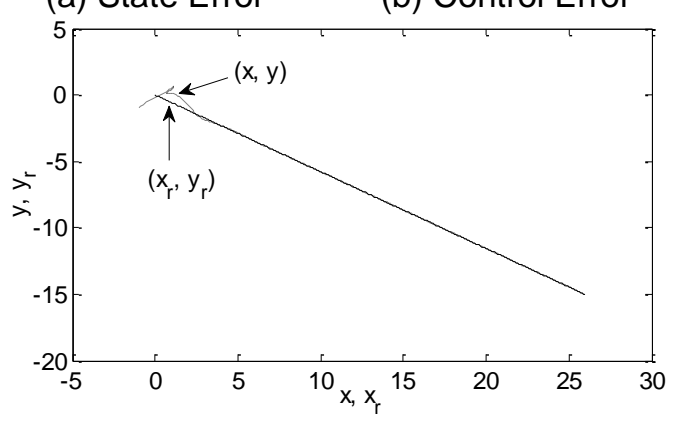

(c) Geometrical Locus

Figure 1. Straight Line Tracking Simulation Time 30 Seconds

It seems that the peaking phenomenon does not appear in Figure 1, in fact that is wrong. Only when $V(t)$ is closed to zero, the peaking phenomenon will appear. The larger the $k_{1}$ is, the sooner peaking phenomenon appears; When $k_{1}$ is constant, the peaking phenomenon will appear finally as long as the simulation time is long enough.

\subsection{Circumference Tracking}

Target trajectory is the circumference of radius 1, using the controller that mentioned in Theorem 2. The results of the simulation are shown in Figure 2. From Figure 2(b), the severe peaking phenomenon is appeared at about 27 seconds. In Figure 2(a) there is peaking phenomenon too. Therefore, state error can't really converge to zero, asymptotic exponential convergent properties cannot be achieved practically. 


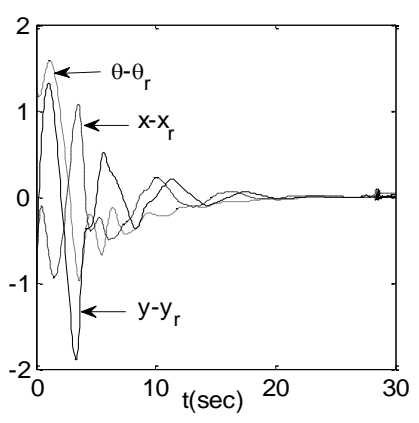

(a) State Error

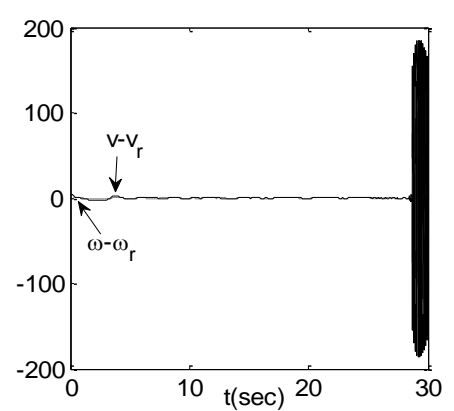

(b) Control Error

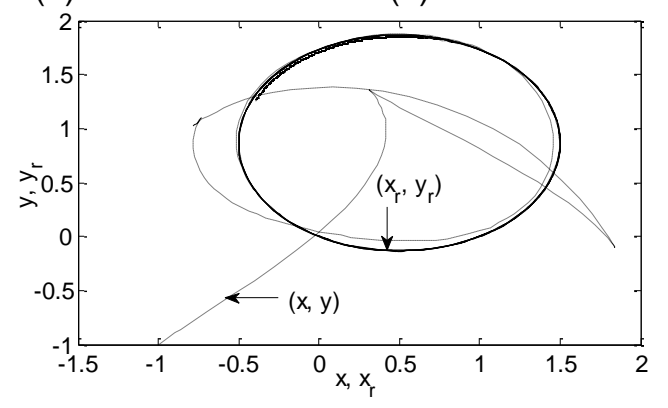

(c) Geometrical Locus

Figure 2. Circumference Tracking

\subsection{The Universal Practical Controller}

The universal practical controller is mentioned in Theorem 2, simulations on straight line tracking and circular trajectory are shown in Figure 3 and Figure 4.

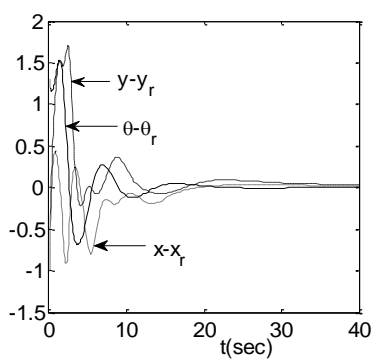

(a) State Error

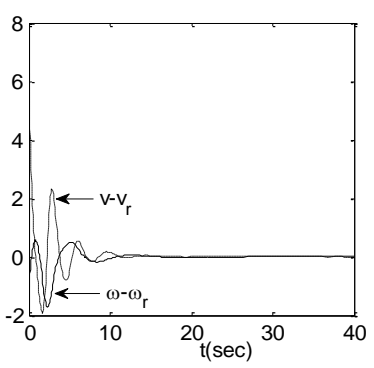

(b)Control Error

Figure 3. The Practical Tracking Straight Trajectory

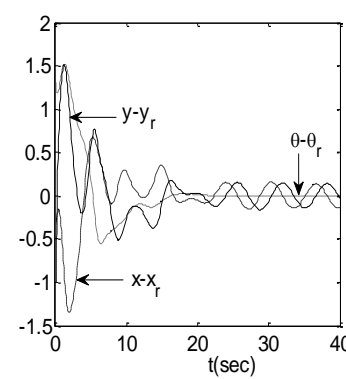

(a) State Error

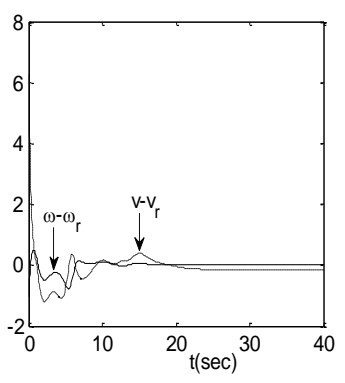

(b) Control Error

Figure 4. The Practical Tracking Circular Path 
The simulation time of Figure 3 and Figure 4 is the same as Figure 1 and Figure 2,but there is no peaking phenomenon in Figure 3 and Figure 4. This shows that the peaking phenomenon is eliminated according to theorem 3. However, the state error curve of Figure 3 and Figure4 doesn't converge to zero, but converge to a small neighborhood of zero.

In addition, in Figure 3 and Figure 4, other control error exponential converge to zero expect $v-v_{r}$ in Figure 4 . It seems to be inconsistent with the conclusion of Theorem 2. The reason is the transformation from robot coordinate system $(x, y, \theta, v, \omega)$ and threedimensional chain coordinate system $\left(x_{1}, x_{2}, x_{3}, u_{1}, u_{2}\right)$. Considering the coordinate transformation (21), Theorem 2 shows that $\left(u_{1}, u_{2}\right)$ will exponential converge to $\left(u_{1 r}, u_{2 r}\right)$ so $\left(\omega, v-\omega x_{3}\right)$ will exponential converge to $\left(\omega_{r}, v_{r}-\omega_{r} x_{3 r}\right)$. In Figure $3, \omega_{r}=0$,so $v \rightarrow v_{r}$.In Figure $4, \omega_{r}$ is a nonzero constant, and ${ }^{x_{3}}$ can only converge to the neighbor of ${ }^{x^{3 r}},{ }^{v}$ can only converge to $v_{r}$ as well.

\section{Conclusion}

For the first order nonholonomic system, paper studies three universal controller based on dynamic feedback, realizes the exponential of convergence, asymptotic stability and the practical stability respectively, which can be used to point stabilization and trajectory tracking at the same time without modifying. Detailed simulations on the three-dimension chained system are carried out, and the results show the effectiveness of the proposed controllers.

\section{Acknowledgements}

This work is financially supported by The National Natural Science Foundation of China (Grant No. 51404073), The National Natural Science Foundation of China (Grant No.51574088), Support from the University Nursing Program for Young Scholars with Creative Talents in Heilongjiang Province (Grant No. UNPYSCT-2016084), The 9th special China Postdoctoral science Foundation projects (Grant No.2016T90268), China Postdoctoral Foundation (Grant No.2014M550180), Hei Long Jiang Postdoctoral Foundation (Grant No.LBH-TZ-0503), The Scientific Research Fund of Heilongjiang Provincial Department of Education (Grant No.12541090). Northeast petroleum university graduate student innovation research projects (Grant No.YJSCX2016010NEPU)

\section{References}

[1] P. Frihauf, S. J. Liu and K. Miroslav, "A single forward-velocity control signal for stochastic source seeking with multiple nonholonomic vehicles", Journal of Dynamic Systems, Measurement and Control, vol. 5, no. 136, (2014).

[2] A. Sakly, "Stability and stabilization studies for a class of switched nonlinear systems via vector norms approach", ISA transactions, vol. 2, no. 31, (2014).

[3] J. Yin, "Asymptotic stability in probability and stabilization for a class of discrete-time stochastic systems", International Journal of Robust and Nonlinear Control, vol. 15, no. 25, (2015).

[4] K. D. Do, "Practical control of under actuated ships", Ocean Engineering, vol. 13, no. 37, (2010).

[5] K. D. Do, Z. P. Jiang and J. Pan, "Simultaneous tracking and stabilization of mobile robots: an adaptive approach", IEEE Transactions on Automatic Control, vol. 7, no. 49, (2004).

[6] W. E. Dixon, D. M. Darken and F. M. Zhang, "Global exponential tracking control of a mobile robot system via a PE condition", IEEE Transactions on System. Man and Cybernetics-Part B: Cybernetics, vol. 1, no. 30, (2000).

[7] G. Oriolo, A. De Luca and M. Vendittelli, "WMR control via dynamic feedback linearization: design, implementation, and experimental validation", IEEE Transactions on Control Systems Technology, vol. 6 , no. $10,(\mathbf{2 0 0 3})$. 
[8] T. C. Lee, K. T. Song and H. C. Lee, "Tracking control of unicycle-modeled mobile robots using a saturation feedback controller", IEEE Transactions on Control Systems Technology, vol. 2, no. 9, (2001).

[9] B. J. Li, H. Chen and J. F. Chen, "Global finite-time stablization for a class of nonholonomic chained system with input saturation", Journal of Information and Computational Science, vol. 3, no. 11, (2014).

[10] E. P. Li, L. J. Long and J. Zhao, "Global output-feedback stabilization for a class of switched uncertain nonlinear systems", Applied Mathematics and Computation, vol. 1, no. 256, (2015).

[11] F. Z. Gao, F. S. Yuan and Y. Q. Wu, "Adaptive stabilization for a class of stochastic nonlinearly parameterized nonholonomic systems with unknown control coefficients", Asian Journal of Control, vol. 6 , no. 16, (2014).

[12] K. D. Do, "Global inverse optimal stabilization of stochastic nonholonomic", Systems and Control Letters, vol. 4, no. 75, (2015).

[13] Y. N. Wang, Z. Q. Miao, H. Zhong and Q. Pan, "Simultaneous stabilization and tracking of nonholonomic mobile robots: A Lyapunov-based approach", IEEE Transactions on Control Systems Technology, vol. 4, no. 23, (2015).

[14] C. Samson, "Control of chained systems-application to path following and time-varying point stabilization of mobile robots", IEEE Transactions on Automatic Control, vol. 1, no. 40, (1995).

[15] K. D. Do and Z. P. Jiang, "Tracking of underactuated ships", Systems \& Control Letters, vol. 4, no. 47, (2002).

[16] B. L. Ma and S. K. Tso, "Unified controller for both trajectory tracking and point regulation of secondorder nonholonomic chained systems", Robotics and Autonomous Systems, vol. 4, no. 56, (2008).

[17] J. H. Li, P. M. Lee and B. H. Jim, "Point-to-Point navigation of under-actuated ships", Automatica, vol. 12 , no. 44, (2008).

[18] W. E. Dixon, D. M. Dawson and F. Zhang, "Global exponential tracking control of a mobile robot system via a PE condition", IEEE Transactions on Systems, Man, and Cybernetics, Part B:Cybernetics, vol. 1, no. 30, (2000).

[19] D. Lapierre and S. A. Pascoal, "Nonsingular path following control of a unicycle in the presence of parametric modeling uncertainties", International Journal of Robust and Nonlinear Control, vol. 10, no. 16, (2006).

[20] M. A. Lashari, Q. Zia and M. Rehan, "Stabilization and tracking control for a class of discrete-time nonlinear systems", International Bhurban Conference on Applied and Science \& Technology, Islamabad, Islamic Republic of Pakistan, (2015).

[21] H. Z. Xiao, Z. J. Li and y. Chun, "Stabilization of nonholonomic chained systems via model predictive control", IEEE 2014 International Conference on Multisensor Fusion and Integration for Intelligent Systems, Beijing, China, (2014).

[22] X. Wenjing and M. Baoli, "Adaptive set stabilization of wheeled mobile robot with uncertainties", The 29th Chinese Control Conference, Beijing, China, (2010).

\section{Author}

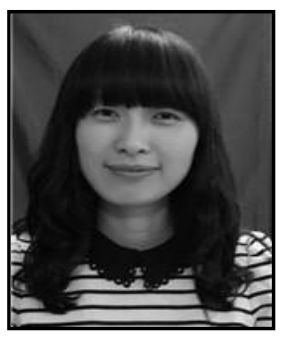

Ting-ting Wang, was born on January 22, 1982 in Anda, Heilongjiang, China. She received the B. A. and M. A. degrees in 2004 and 2007, respectively, all from Daqing Petroleum Institute, China. From 2007 to 2009 she was Assistant at School of Electrical Engineering \& Information, Daqing Petroleum Institute, China. From 2009 to 2014 she was Lecturer at School of Electrical Engineering \& Information, Northeast Petroleum University, China. Since 2014 she is Associate Professor at School of Electrical Engineering \& Information, Northeast Petroleum University, China. Her scientific interests include aspects on information and control. 


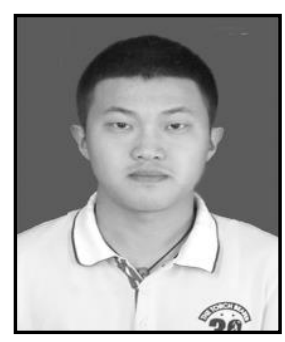

Tian-you Li, was born on February 22, 991 in QiQihar, Heilongjiang, China. He received the university education in 2010 in Northeast university of petroleum China. He majored in Automation. He got his Bachelor's degree in 2014. Since 2015, he began to receive postgraduate education at School of Electrical Engineering \& Information, Northeast Petroleum University, China. His research direction is control science and engineering.

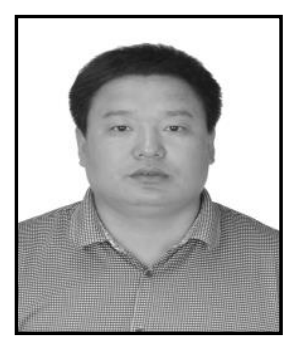

Wan-chun Zhao, was born December 29,1978 in Jiutai, Jilin, China. He received the B. A., M. A. and Ph.D degrees in 2002 and 2005 and 2009, respectively, all from Daqing Petroleum Institute, China. From 2009 to 2010 he was Lecturer at School of Petroleum Engineering, Daqing Petroleum Institute, China. From 2010 to 2013 he was Associate Professor at School of Petroleum Engineering, Northeast Petroleum University, China. Since 2013 he is Professor at School of Petroleum Engineering, Northeast Petroleum University, China. Her scientific interests include aspects on information and control. 